\title{
Proximity Effect and Multiple Andreev Reflections in Gold Atomic Contacts
}

\author{
E. Scheer, ${ }^{1, *}$ W. Belzig, ${ }^{2}$ Y. Naveh, ${ }^{3, \dagger}$ M. H. Devoret, ${ }^{4}$ D. Esteve, ${ }^{4}$ and C. Urbina ${ }^{4}$ \\ ${ }^{1}$ Physikalisches Institut, Universität Karlsruhe, D-76128 Karlsruhe, Germany \\ ${ }^{2}$ Department of Applied Physics, Delft University of Technology, 2600 GA Delft, The Netherlands \\ ${ }^{3}$ Department of Physics, State University of New York at Stony Brook, Stony Brook, New York 11794 \\ ${ }^{4}$ Service de Physique de l'Etat Condensé, Commissariat à l'Energie Atomique, Saclay, F-91191 Gif-sur-Yvette Cedex, France
}

(Received 9 March 2000)

\begin{abstract}
We investigate the electronic transport properties of gold point contacts with superconducting aluminum leads. The modifications induced by the proximity effect in the quasiparticle density of states at the contact region are measured by tunnel spectroscopy. The theory of transport through multiple Andreev reflections is extended to incorporate these effects and used to determine the number and transmission coefficients of the conduction channels in the contact regime. We find that the smallest contacts, formed by one gold atom between the electrodes, contribute one single channel to the transport with variable transmission $T$ between 0.1 and 1 .
\end{abstract}

DOI: $10.1103 /$ PhysRevLett.86.284

PACS numbers: 73.40.Jn, 73.20.Dx, 74.50. $+\mathrm{r}$

During the last decade, superconductivity has been probed at length scales shorter than the quantum coherence length of electron transport in the normal state. In this new field called "mesoscopic superconductivity" [1], Andreev reflection has emerged as a central concept [2], that applies at all scales, because it fits naturally in the independent conduction channels description of mesoscopic conductors [3]. Indeed, the reflection of quasielectrons into quasiholes induced by a pairing potential arises within each channel. An equilibrium phenomenon like the proximity effect (PE), which corresponds to the dilution of the superconducting order in a normal metal in contact with a superconducting one, is now well understood in terms of Andreev reflections in all channels at the interface. A nonequilibrium phenomenon like the dissipative transport between two superconducting electrodes connected through a small number of conduction channels is well understood in terms of coherent Andreev reflections at both electrodes [4]. Indeed, it has been shown experimentally [5] that the current through atomic-size contacts obeys with great accuracy the full quantum theory of coherent multiple Andreev reflections (MAR) [6-9]. What happens when both aspects are present simultaneously? More precisely, how are MAR affected when the two electrodes are not intrinsic, BCS-like superconductors, but are instead PE superconductors? This is the question we address in this Letter both experimentally and theoretically.

The experiments are carried out on atomic-size contacts between two small gold electrodes in which the PE is induced by large aluminum reservoirs. We produce the contacts by means of microfabricated break junctions [10]. Using shadow evaporations through a suspended mask, we fabricate two $\mathrm{Al}$ reservoirs of thickness $d_{\mathrm{A} 1}$ separated by a gap of width $2 L_{N}$. Without breaking the vacuum, two Au layers of thickness $d_{\mathrm{Au}} / 2$ are evaporated at two different angles in order to fill the gap and to form a continuous film with a narrow constriction in the middle (see Fig. 1). The central part is suspended over $2 \mu \mathrm{m}$ by selective etching of a sacrificial polyimide layer.

The bridges thus formed are broken at the constriction by controlled bending of the elastic substrate, at very low temperatures $\Theta<1 \mathrm{~K}$ and under cryogenic vacuum conditions. After measurement, we check in a scanning electron microscope (SEM) that the $\mathrm{Al}$ electrodes are well separated and that the rupture of the contact had occurred at the Au region (cf. Fig. 1c). As in previous experiments [11], as the bridges are stretched, their conductance $G$ decreases in a series of steps of the order of the conductance quantum $G_{0}=2 e^{2} / h$, separated by plateaus with smaller steps (cf. Fig. 4). The breaking is indicated by the onset of an exponential decrease of $G$ with the distance between the electrodes, a hallmark of the tunneling regime. The last contact before the break, which possibly corresponds to a single atom contact, has usually a conductance below $G_{0}$.

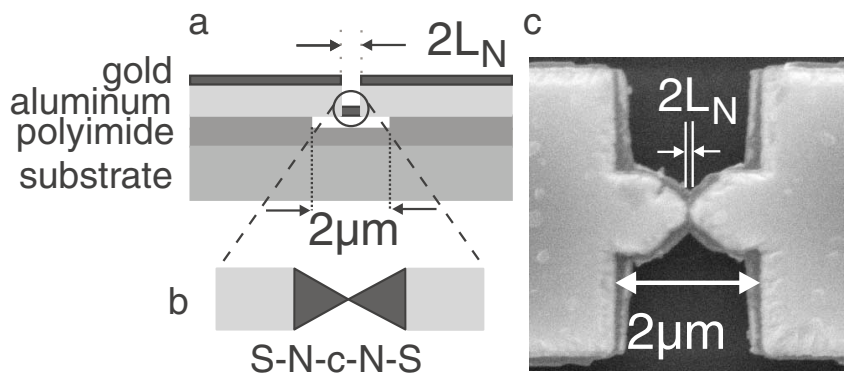

FIG. 1. (a) Schematic side view of the samples. The metallic thin films are evaporated on top of a flexible substrate covered by a polyimide sacrificial layer. The latter is etched away to suspend the central bridge that connects the two large Al electrodes through a small Au constriction. (b) Schematic top view of the central part of the resulting system. Two nominally identical NS structures coupled through a constriction which can be controlled in situ down to the atomic size. (c) SEM micrograph of sample No. 1 (top view). The Al electrodes are the brightest regions. The narrow and dark skirt around them corresponds to the gold layer, through which the contact is established. 
The technique allows one to stabilize contacts and tunnel configurations over a wide range of conductances.

Figure 2 shows current-voltage characteristics (IVs) measured in the tunneling regime, at $\Theta \leq 50 \mathrm{mK}$, for two Al-Au-Al (No. 1 and No. 2) and one pure Al sample (No. 3), which displays the well-known current rise at twice the voltage corresponding to the gap energy $\Delta=$ $180 \mu \mathrm{eV}$. A similar rise at $2 E_{g}<2 \Delta$ is observed for both $\mathrm{Al}-\mathrm{Au}-\mathrm{Al}$ samples. The values of the induced gap $E_{g}$ in Table I have been determined through best fits of the $I V \mathrm{~s}$, assuming BCS superconductors. Also noticeable is the development of a maximum of the current, i.e., a region with negative differential conductance $d I / d V$, slightly above $2 E_{g}$. Above the critical temperature (or the critical field) given in Table I all these well-known signatures of the proximity effect disappear and the $I V \mathrm{~s}$ become linear. The appearance of an induced gap in the quasiparticle density of states (DOS) $\rho(E)$ of diffusive superconductor-normal metal-superconductor (SNS) structures has been predicted $[13,14]$ and found experimentally before $[15,16]$. The maximum in the current has also been observed in large proximity effect tunnel junctions [17] and stems from the deviations of $\rho(E)$ from the BCS spectrum [18].

In order to describe these $I V \mathrm{~s}$, we calculate both the spatial dependence of $\rho(E, x)$ ( $x$ is the transport direction) and the size of the induced gap using a one-dimensional diffusive model. The sample geometry is approximated by two identical NS structures, weakly coupled at the $\mathrm{N}$ side via an opaque tunnel barrier with transmission $T$. This implies that the DOS at the tunnel barrier can be calculated

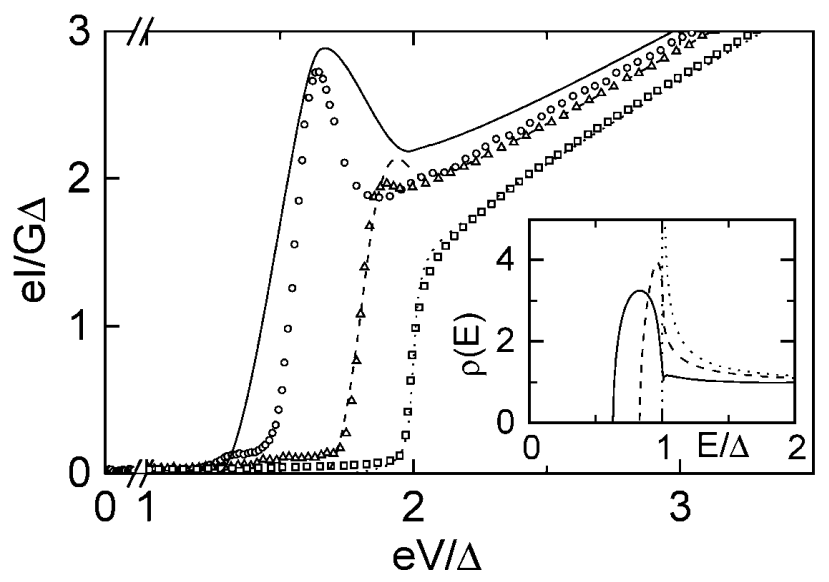

FIG. 2. Measured (symbols) and calculated (lines) $I V \mathrm{~s}$ in the tunnel regime for Al-Au-Al samples No. 1 (triangles) and No. 2 (circles), and pure Al sample No. 3 (squares). The dotted line is the $I V$ calculated for pure $\mathrm{Al}$ at $\Theta \leq 50 \mathrm{mK}$ using BCS theory. The other lines are fits of the $I V \mathrm{~s}$ for samples No. 1 (dashed line) and No. 2 (solid line) according to the theory of Belzig et al. [12] with the parameters given in Table I. The voltage axis is normalized to the measured superconducting gap of Al $\Delta / e=180 \mu \mathrm{V}$. The current axis is normalized to $G \Delta / e$, where $G$ is the conductance measured by the slope of the $I V \mathrm{~s}$ far above the gap. Inset: Density of states for BCS and proximity superconductors calculated with the best-fits parameters of the $I V \mathrm{~s}$ on the main panel. neglecting the presence of the second NS structure (see Fig. 1b). The spatial and energy dependence of the superconducting pair correlations are described by two retarded Greens functions $G(E, x)$ and $F(E, x)$ which are evaluated numerically [12]. The DOS is then given by $\rho(E, x)=$ $\rho_{0} \operatorname{Re}\{G(E, x)\}$, where $\rho_{0}$ is the DOS in the normal state. The superconductor is assumed to be infinite and the length of the normal metal to be $L_{N}$. The second important parameter in the model is the "mismatch parameter" $\Gamma=$ $\sigma_{\mathrm{Au}} / \sigma_{\mathrm{Al}}\left(D_{\mathrm{Al}} / D_{\mathrm{Au}}\right)^{1 / 2}$, where $\sigma$ and $D$ are, respectively, the conductivity and the diffusion constant of each metal. It accounts for the difference of the Fermi velocities and disorder of the two metals in the normal state, leading to an effective barrier for the electrons. Both parameters can be estimated from the sample geometry (see Table I) and the elastic mean free path of the films. From the residual resistance ratio $\mathrm{RRR}=R(\Theta=300 \mathrm{~K}) / R(\Theta=4 \mathrm{~K})$ of our Al films, we estimate the elastic mean free path, $l_{\mathrm{Al}} \approx 65 \mathrm{~nm}$, the diffusion constant $D_{\mathrm{Al}}=v_{F} l_{\mathrm{Al}} / 3=$ $0.042 \mathrm{~m}^{2} / \mathrm{s}$, and the diffusive superconducting coherence length $\xi_{S}=\left(\hbar D_{\mathrm{Al}} / 2 \Delta\right)^{1 / 2}=280 \mathrm{~nm}$. For the Au layers RRR is typically around 1.5 to 1.9 , corresponding to an elastic mean free path of $l_{\mathrm{Au}} \approx 25-45 \mathrm{~nm}$.

The tunnel $I V \mathrm{~s}$ are calculated by autoconvoluting the DOS at the contact, i.e., at $x=L_{N}$. The $\rho\left(E, L_{N}\right)$ and $I V$ s calculated using the best-fit parameters of Table I are displayed in Fig. 2. For comparison we also show the respective quantities for a tunnel junction between $\mathrm{BCS}$ superconductors (pure $\mathrm{Al}$ sample). Considering the simplicity of the model geometry and the fact that since $L_{N} \sim l_{\mathrm{Au}}$ the samples are at the borderline of the validity of the diffusive transport theory, the calculated and experimental $I V \mathrm{~s}$ are in reasonable agreement.

When the two electrodes are brought back into contact the $I V \mathrm{~s}$ show the characteristic subgap structure due to MAR [19]. Figure 3 displays several $I V$ s measured on contacts corresponding to the last conductance plateau before breaking, for samples No. 1 and No. 2. The conductances of all examples are smaller than $G_{0}$. For small conductances, there are maxima in the $I V$ at $2 E_{g} / e$ and at the submultiples $2 E_{g} / m e(m=2,3, \ldots)$. This indicates that the modifications induced on the DOS by the PE are still present in the contact regime.

In order to describe these contact $I V \mathrm{~s}$ we generalize the scattering theory of MAR of Averin and Bardas [7]. The proximity effect does not only modify the DOS but also the Andreev-reflection amplitude according to [20,21]

$$
A\left(E, L_{N}\right)=-i \frac{F\left(E, L_{N}\right)}{1+G\left(E, L_{N}\right)} .
$$

We introduce this amplitude into the scattering formalism of Ref. [7] and calculate the $I V$ s for arbitrary transmission. This assumption implies that, as for the DOS, the Andreev-reflection amplitude $A\left(E, L_{N}\right)$ at the tip of each electrode is not altered when bringing the two electrodes into contact through a small number of conduction channels. 
TABLE I. Sample number, thickness of the Al (Au) layer $d_{\mathrm{Al}(\mathrm{Au})}$, elastic mean free path of the Au layer $l_{\mathrm{Au}}$, minigap $E_{g}$ (superconducting gap $\Delta$ for sample No. 3), critical temperature $\Theta_{c}$, critical field $B_{c}$, spacing between Al electrodes as deduced by best fit to the theory of Ref. [12] $\left(L_{N} / \xi_{S}\right)_{\text {fit }}$ (assuming $\left.\xi_{S}=280 \mathrm{~nm}\right)$ and as estimated from the micrographs $\left(L_{N} / \xi_{S}\right)_{\text {exp }}$, and best-fit value $\Gamma_{\text {fit }}$ and estimated value $\Gamma_{\exp }$ of the mismatch parameter.

\begin{tabular}{|c|c|c|c|c|c|c|c|c|c|c|}
\hline \multirow[b]{2}{*}{ No. } & \multirow{2}{*}{$\begin{array}{c}d_{\mathrm{Al}} \\
(\mathrm{nm})\end{array}$} & \multirow{2}{*}{$\begin{array}{c}d_{\mathrm{Au}} \\
(\mathrm{nm})\end{array}$} & \multirow{2}{*}{$\begin{array}{c}l_{\mathrm{Au}} \\
(\mathrm{nm})\end{array}$} & \multirow{2}{*}{$\begin{array}{l}E_{g}[\Delta] \\
(\mu \mathrm{eV})\end{array}$} & \multirow{2}{*}{$\begin{array}{c}\Theta_{c} \\
(\mathrm{~K})\end{array}$} & \multirow{2}{*}{$\begin{array}{c}B_{c} \\
(\mathrm{mT})\end{array}$} & \multicolumn{2}{|c|}{$\left(L_{N} / \xi_{S}\right)$} & \multicolumn{2}{|c|}{$\Gamma$} \\
\hline & & & & & & & fit & expt. & fit & expt. \\
\hline 1 & 300 & 20 & 45 & 160 & 1.21 & 5.05 & 0.16 & 0.1 & 0.6 & 0.54 \\
\hline 2 & 400 & 30 & 25 & 140 & 1.21 & 6.35 & 0.8 & 0.4 & 0.2 & 0.4 \\
\hline 3 & 150 & $\cdots$ & $\ldots$ & 180 & 1.21 & 10.2 & $\ldots$ & $\cdots$ & $\ldots$ & $\ldots$ \\
\hline
\end{tabular}

The best-fit $I V$ s calculated within this model assuming a single channel are also shown in Fig. 3. The transmission coefficient which corresponds to the slope of the $I V$ far above the gap is the only fitting parameter. For comparison we also show the corresponding best-fit $I V$ s calculated for pure BCS superconductors with a gap $E_{g}$. Both models give essentially the same transmission coefficient values within $1 \%$. However, for low and medium transmissions the proximity model describes the experimental $I V \mathrm{~s}$ significantly better than the BCS model. The reason is that in this regime the cusps in the $I V$ are most pronounced emphasizing the different behaviors of $A\left(E>E_{g}\right)$ of the two models. For high transmissions $T>0.9$, the differences between proximity and BCS models are smaller, since the shape of $A\left(E, L_{N}\right)$ becomes less important.

In our previous work we had shown that even the smallest contacts of multivalent metals as, e.g., $\mathrm{Al}, \mathrm{Nb}$, or $\mathrm{Pb}$ transmit more than one channel [22]. Opposed to this observation, we find for $\mathrm{Au}$ stable configurations in the contact regime which transmit only a single conduction channel. The transmission of this channel can be varied widely when rearranging the geometry of the central region by opening and closing again the contact. Although infrequently, it is possible to stabilize single-channel contacts with almost perfect transmission. Several authors have predicted, within different approaches including first principles [23], jellium models [24], molecular dynam-

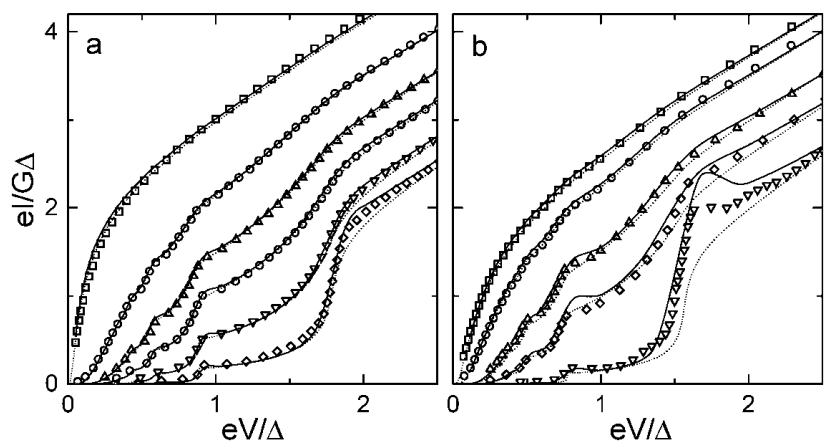

FIG. 3. (a) [(b)] Measured current-voltage characteristics (symbols) of six [five] different contacts of sample No. 1 [No. 2] at $30 \mathrm{mK}$, and best single channel fits for a BCS superconductor with gap $E_{g}$ (dotted lines) and proximity superconductors (solid lines) with parameters as indicated in Table I. The individual channel transmissions obtained from the fits are from top to bottom: $T=0.98,0.85,0.69,0.55,0.35$, and $0.15[T=$ $0.93,0.86,0.68,0.50$, and 0.11$]$. Voltage and current are in reduced units. ics [25], and tight-binding methods [26], that single-atom contacts of monovalent metals should transmit electrons through a single channel. In the latter work, the mechanism which gives rise to a single-channel transport is the formation of a resonance at the Fermi energy. The transmission of this channel is a function of the contact geometry [26]. Transmission close to one is predicted only if the tightbinding coupling rates of the orbitals of the central atom to the right and left electrode $\gamma_{L(R)}$ are equal. Disorder within several atomic layers from the central atom reduces the value of the transmission coefficient, explaining the fact that the conductance of the single-atom contact is often smaller than $G_{0}$ and that it varies when further stretching or pushing the contact [22]. A statistical analysis of $\approx 2000$ $I V$ s of single-atom contacts on four samples yields an average value of $T=0.6 \pm 0.15$, which is much lower than the predicted saturation value for perfect symmetric configuration. Assuming perfect pinning of the resonance to the Fermi level, this result would correspond to an asymmetry of the coupling rates $\gamma_{L} / \gamma_{R} \approx 4$. Another possible explanation of the reduced transmission within the framework of the model of Ref. [26] could be a deviation of the resonance maximum from the Fermi level due to imperfect charge neutrality or reduced screening at the central atom. In earlier experiments on gold single-atom contacts by means of scanning tunneling microscopy techniques, quantized conductance values and well pronounced conductance steps were observed only after repeated mechanical workout of the contact [11]. Since with our break-junction technique it is impractical to perform such a treatment, we only occasionally observe almost saturated transmission values, i.e., perfectly ballistic channels.

By repeatedly recording the $I V \mathrm{~s}$ while slowly opening or closing the bridges, it is possible to follow the development of the channel distribution by applying the analysis procedure described in detail in Ref. [5]. We often observe conductance plateaus with $G \leq G_{0}$, which cannot be described by a single channel, but are correctly fitted when taking into account two or, less frequently, three channels. In Fig. 4 we plot $I / V$ (scaled to the conductance $G$ ) vs voltage for three contacts taken on sample No. 2. In this scaling, the differences between the best fits according to the PE model and BCS theory are clearly visible. As in the single-channel situation, we observe strong MAR features which are better described by the PE model than by the BCS one. Also plotted in Fig. 4 is the best one-channel fit (dash-dotted line) for the bottommost experimental curve. 


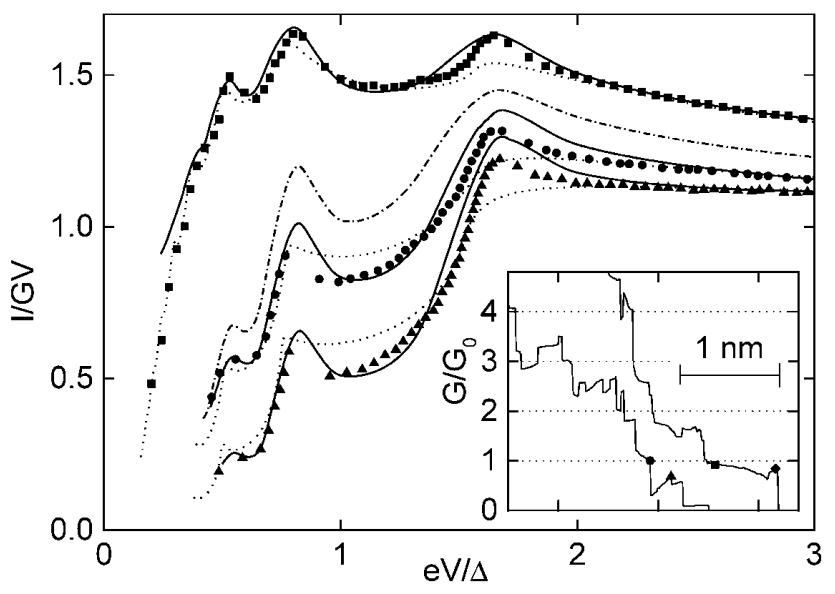

FIG. 4. Measured $I / V$ vs $V$ characteristics (symbols) of three different contacts with $G \leq G_{0}$ for sample No. 2 at $30 \mathrm{mK}$ and best numerical fits for BCS superconductors with gap $E_{g}=$ $140 \mu \mathrm{eV}$ (dotted lines), and with the PE model (solid lines) taking into account two or three channels. Dash-dotted line: best single-channel fit with the PE model to the bottommost curve. The individual channel transmissions obtained from the PE (BCS) fits are squares: $T_{1}=0.76, T_{2}=0.15\left(T_{1}=0.74\right.$, $\left.T_{2}=0.18\right)$; circles: $T_{1}=0.46, T_{2}=0.40, T_{3}=0.14\left(T_{1}=\right.$ $\left.0.58, T_{2}=0.40, T_{3}=0.02\right)$; triangles: $T_{1}=0.31, T_{2}=0.26$ $\left(T_{1}=0.45, T_{2}=0.13\right)$; for the single-channel fit: $T=0.51$. Inset: Evolution of the conductance $G$ as the sample is stretched continuously. The symbols refer to the symbols from the main panel and indicate the particular position at which the $I / V$ vs $V$ curves were recorded. The diamond indicates a single-channel contact with $T=0.86$, whose $I V$ is shown in Fig. 3b. In this "plateau" the number of channels changes from two to one at the minimum at $0.1 \mathrm{~nm}$ before the rupture.

Clearly, the number of channels is a robust outcome of the fitting procedure, even if not all details of the subgap structure can be fitted perfectly. Although the number of channels found with the PE and BCS models is always the same, even for contacts with up to seven channels, the transmission values distribution does change (see figure caption). Note that in repeated opening measurements, as usually performed to calculate the so-called "conductance histograms" [11], all the regions with $G \leq G_{0}$ would contribute to the well-known first conductance peak of gold point contacts although, as we have shown, part of them accommodate several channels.

To summarize, we have presented an investigation of the transport properties of gold tunnel and few-atom contacts having superconducting leads. The current-voltage characteristics in the tunnel regime strongly differ from what is observed for a pure BCS superconductor. From their analysis within the framework of the diffusive proximity effect, we extracted the quasiparticle density of states at the contact region and the corresponding Andreev reflection amplitude. This last ingredient was then used to extend the theory of MAR to the case of a channel with arbitrary transmission between PE superconductors. Although this extended model does not explain quantitatively all the details of the $I V \mathrm{~s}$ of few-atom contacts, which also display deviations from BCS behavior, it does explain the general trends. An important conclusion of this analysis is that the smallest gold contacts observable in the experiments accommodate a single conduction channel in accordance with theoretical predictions for single-atom contacts.

We thank D. V. Averin, C. Bruder, J.C. Cuevas, H. v. Löhneysen, J. M. van Ruitenbeek, G. Schön, and C. Strunk for helpful discussions. This work was partially supported by the French BNM and the German DFG through a research grant and SFB 195.

*Present address: Fachbereich Physik, Universität Konstanz, D-78457 Konstanz, Germany.

Electronic address: Elke.Scheer@uni-konstanz.de

${ }^{\dagger}$ Present address: IBM Research Labs in Haifa, MATAM, Haifa 31905, Israel.

[1] See, e.g., special issue on Mesoscopic Superconductivity, edited by P.F. Bagwell [Superlattices Microstruct. 25, Nos. 5-6 (1999)].

[2] B. Pannetier and H. Courtois, J. Low Temp. Phys. 118, 599 (2000).

[3] R. Landauer, IBM J. Res. Dev. 1, 223 (1957); Philos. Mag. 21, 863 (1970).

[4] G.E. Blonder, M. Tinkham, and T.M. Klapwijk, Phys. Rev. B 25, 4515 (1982).

[5] E. Scheer et al., Phys. Rev. Lett. 78, 3535 (1997).

[6] L. B. Arnold, J. Low Temp. Phys. 68, 1 (1987).

[7] D. Averin and A. Bardas, Phys. Rev. Lett. 75, 1831 (1995).

[8] J. C. Cuevas, A. Martín-Rodero, and A. Levy Yeyati, Phys. Rev. B 54, 7366 (1996).

[9] E. N. Bratus et al., Phys. Rev. B 55, 12666 (1997).

[10] J. M. van Ruitenbeek et al., Rev. Sci. Instrum. 67, 108 (1996).

[11] See, e.g., J. M. van Ruitenbeek, in Mesoscopic Electron Transport, edited by L. L. Sohn, L. P. Kouwenhoven, and G. Schön, NATO ASI, Ser. E, Vol. 345 (Kluwer Academic Publishers, Dordrecht, 1997).

[12] W. Belzig, C. Bruder, and G. Schön, Phys. Rev. B 54, 9443 (1996).

[13] A. A. Golubov et al., Phys. Rev. B 55, 1123 (1997).

[14] F. Zhou et al., J. Low Temp. Phys. 110, 841 (1998).

[15] C. J. Adkins and B.W. Kington, Phys. Rev. 177, 777 (1969).

[16] S. H. Tessmer et al., Phys. Rev. Lett. 70, 3135 (1993).

[17] A. Zehnder et al., Phys. Rev. B 59, 8875 (1999).

[18] MAR processes at the $\mathrm{Au}-\mathrm{Al}$ interfaces which would give rise to nonlinearities at $V=2 \Delta / e$ are not observable because of the negligible interface resistance.

[19] M. Octavio et al., Phys. Rev. B 27, 6739 (1983).

[20] B.A. Aminov, A. A. Golubov, and M. Yu. Kupriyanov, Phys. Rev. B 53, 365 (1996).

[21] A. V. Zaitsev and D. V. Averin, Phys. Rev. Lett. 80, 3602 (1998).

[22] E. Scheer et al., Nature (London) 394, 154 (1998).

[23] N. D. Lang, Phys. Rev. B 36, 8173 (1987).

[24] C. A. Stafford et al., Phys. Rev. Lett. 79, 2863 (1997).

[25] M. Brandbyge et al., Phys. Rev. B 56, 14956 (1997).

[26] A. Levy Yeyati, A. Martín-Rodero, and F. Flores, Phys. Rev. B 56, 10369 (1997). 